\title{
Improved method for computing nucleon strangeness
}

\author{
Walter Freeman* \\ University of Arizona \\ E-mail: wfreemanephysics.arizona.edu \\ Doug Toussaint \\ University of Arizona \\ E-mail: doug@physics.arizona.edu
}

\begin{abstract}
The strange quark content of the nucleon, $\langle N|\bar{s} s| N\rangle-\langle 0|\bar{s} s| 0\rangle$, as well as other matrix elements, can be calculated on the lattice by examining correlations between the nucleon propagator and the quark condensate. The largest contribution to statistical error comes from fluctuations in the condensate far from the propagation region that contribute only noise. We will report on a technique for considering only the condensate near the propagation region, significantly reducing the statistical error.
\end{abstract}

The XXVIII International Symposium on Lattice Field Theory

June 14-19,2010

Villasimius, Sardinia Italy

\footnotetext{
*Speaker.
} 


\section{Motivation}

The strange quark content of the nucleon is difficult to measure experimentally but is a quantity of wide interest. In particular, the interaction cross section between some proposed dark matter candidates (for instance, neutralinos) and ordinary matter may have a large contribution from Higgs-like exchange with sea strange quarks in the nucleon[1,2]. Specifically, the interest is in the quantity $\left\langle N\left|\int d^{3} x \bar{s} s\right| N\right\rangle-\left\langle 0\left|\int d^{3} x \bar{s} s\right| 0\right\rangle$ - the connected part of the strange quark condensate, integrated over all space, in the nucleon. Recently this quantity has been calculated on the lattice by several groups $[3,4,5,6,7,8]$ using a variety of approaches. Several other results were also presented at Lattice 2010 [9, 10, 11, 12]. These calculations all have large statistical error, owing to the inherently noisy nature of evaluating disconnected diagrams. Our previous calculation using the large MILC library of 2+1 flavor Asqtad staggered fermion configurations [13] found $\langle N|\bar{s} s| N\rangle=0.69(7)_{\text {stat }}(9)_{\text {sys. }}$. Reduction of this large statistical error is thus a priority, and might also make possible better control of systematics; in particular, it may enable a reduction in the systematic error estimate due to excited state pollution of the propagators.

\section{Partial condensate method for reducing statistical error}

In our previous method [3], the matrix element $\langle N|\bar{s} s| N\rangle-\langle 0|\bar{s} s| 0\rangle$ is equated to $\frac{\partial M_{N}}{\partial m_{s}}$ via the Feynman-Hellman theorem. This can then be written as the product of two other partial derivatives $\sum_{t} \frac{\partial M_{N}}{\partial P(t)} \frac{\partial P(t)}{\partial m_{s}}$, where $P(t)$ is the nucleon propagator and the sum runs over a range of propagator separations $t$. The minimum distance used in this range should be chosen large enough that excited states have mostly decayed away but small enough that the signal-to-noise ratio is large; the result is relatively insensitive to the maximum distance because of the rapidly declining signal-to-noise ratio in $P(t)$. Since $M_{N}$ is a complicated function of $P(t)$ defined implicitly by a fitting procedure, the first of these two partial derivatives can be measured most easily by applying small changes to $P(t)$ and examining the resulting change in $M_{N}$. The second of these can be written, using the Feynman-Hellman theorem (in reverse), as $\left\langle P(t) \int d^{4} x \bar{s} s\right\rangle-\langle P(t)\rangle\left\langle\int d^{4} x \bar{s} s\right\rangle$. This is expedient, since the whole-lattice condensate $\int d^{4} x \bar{s}$ has been previously measured by MILC on all of the lattice configurations, as have the propagators $P(t)$. Thus, this method allows a high-statistics calculation of the nucleon strangeness with no additional expenditure of computer time.

However, a major contribution to the statistical error in this calculation comes from fluctuations in the quark condensate that have no physical correlation with the hadron propagator. While the correlation between these fluctuations and the propagator averages to zero in the limit of infinite statistics, with finite statistics they do not, and spurious correlations of this sort are a major contributor to statistical error. The time length of the lattice is much greater than the actual region over which the propagator is measured; for instance, on many of the $a=0.12 \mathrm{fm}$ ensembles, the lattice has a temporal extent of $64 a$, but the longest two-point function used only has a length of $15 a$.

Since there is no physical reason that fluctuations in the quark condensate far from the propagator should be correlated with it, those fluctuations contribute only noise and can be discarded 
without introducing bias; in other words, we replace

$$
\left\langle P(t) \int d^{4} x \bar{s} s\right\rangle-\langle P(t)\rangle\left\langle\int d^{4} x \bar{s} s\right\rangle
$$

with

$$
\left\langle P(t) \int d^{3} x \int_{t_{1}}^{t_{2}} d t \bar{s} s\right\rangle-\langle P(t)\rangle\left\langle\int d^{3} x \int_{t_{1}}^{t_{2}} d t \bar{s} s\right\rangle
$$

where $t_{1}$ and $t_{2}$ are chosen sufficiently far from the propagation region so that they do not affect the final result.

By recalculating the quark condensate and independently storing the value of $\bar{s} s$ on each timeslice, it is possible to use only those timeslices which have a meaningful correlation with the propagator, thus reducing noise and statistical error. It is possible that this reduction in error will also lead to greater precision in the determination of the dependence of $\frac{\partial M_{N}}{\partial m_{s}}$ on the minimum distance used in the fit, allowing for a reduction in the estimate for the statistical error from excited states.

\section{New calculations}

This also requires recalculation of the hadron propagators themselves. In order to make most efficient use of the lattice, MILC uses the average of multiple sources, each located on a different timeslice, to calculate the propagator; in addition, the forward and backward propagators for each source are averaged together. Only this average is stored, but since it contains contributions from the entire time extent of the lattice, it is correlated with the condensate on every timeslice. By recomputing the hadron propagators and saving a separate propagator in each direction for each source timeslice, it is possible to extract only the physically-meaningful correlations.

In the previous calculation, the fit was mostly controlled by the coarsest $(a=0.12 \mathrm{fm})$ data; this is due both to higher statistics of the coarser lattice ensembles and the extra noise from the high-momentum modes present in the finer lattices. Since it is significantly more expensive to recompute the nucleon propagators in this manner on finer lattices, and since the coarser lattices dominate the fit in any case, we have only done this procedure on the $a=0.12 \mathrm{fm}$ lattices. Four of the five ensembles have been completed, and a fifth is in progress.

\section{Validity checking}

A larger reduction in statistical error is possible if a smaller range of condensate around the propagator is kept, but if too much of the condensate is discarded, it is possible that some physically-meaningful correlations will be eliminated along with the noise. It is instructive to look at the correlation between $\int d^{3} x \bar{s} s(t)$ and a propagator with source at $t=0$ and sink at $t=T$; we expect this correlation to fall off rapidly outside the region of propagation from 0 to $T$. Figure 1 shows the correlation between this propagator and the strange quark condensate on different timeslices.

The most notable feature of these plots is the fact that the correlation between the nucleon propagator and the quark condensate is only distinguishable from zero in a narrow range around the propagation region for the two short-distance propagators. 

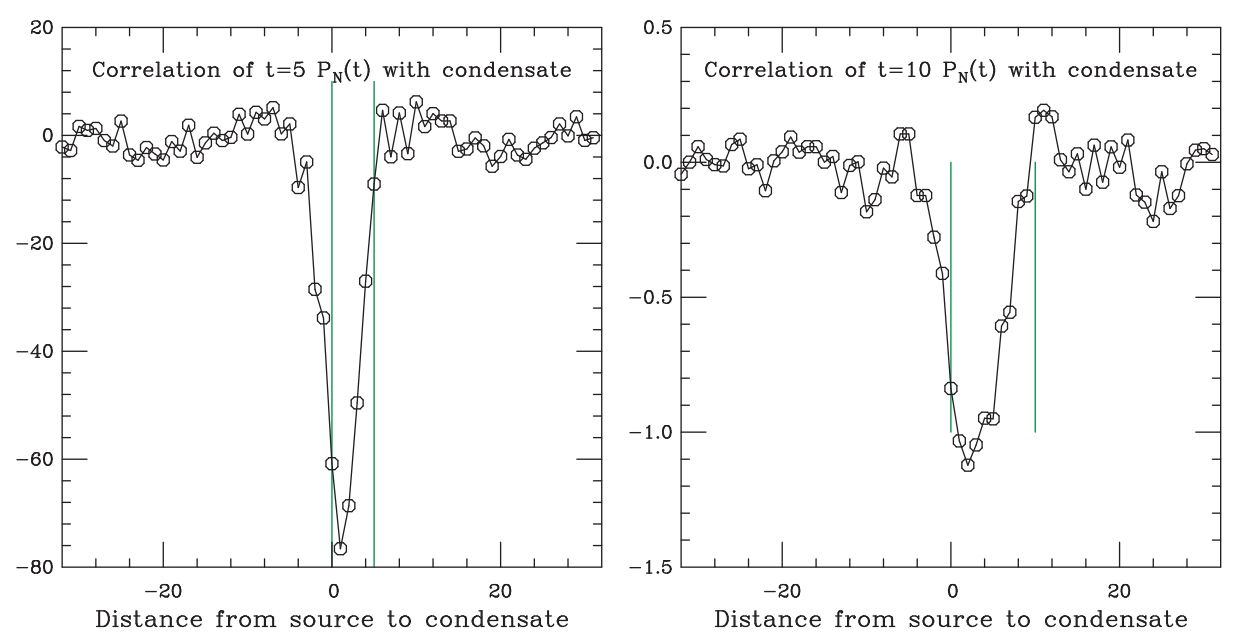

Figure 1: The correlation between nucleon propagators of length $5 a$ and $10 a$ with the strange quark condensate on different timeslices on one of the MILC $a=0.12 \mathrm{fm}$ ensembles. The source and sink of the propagators are marked in green.

These data suggest that it is reasonable to exclude condensate measurements more than a certain padding distance $T_{p}$ from the propagation region; they suggest that this distance is in the vicinity of $4-5 a$. However, in order to truly understand the effect that discarding much of the quark condensate has on the result, it is necessary to examine the dependence of $\frac{\partial M_{N}}{\partial m_{s}}$ itself on the padding size. This dependence is shown in Figure 2.

Note that results using similar padding sizes are highly correlated, since they are based on almost the same data set. The most significant effect of this method is the reduction in statistical error by almost half when using small padding sizes, as expected. While most of the fluctuations in the central value seen here are consistent with statistical effects, for very small pad sizes a sharp downward trend is noticeable in the results. Since using a pad size that is too small amounts to discarding physically significant correlations, the depressed result for low pad sizes is consistent with the introduction of bias.

From examining the average of all four ensembles, shown in Figure 3, it is reasonable to conclude that using a pad size of $4 a$ introduces no obvious bias and greatly reduces the statistical error. The shift in the central value is three percent, consistent with the improvement in the statistical error. Thus it is very unlikely that the systematic error introduced by this method is larger than that; a more realistic upper bound on the systematic error from this procedure is one percent.

\section{Effect on systematic error estimates}

The improved statistical error from this procedure should make improved estimates of the systematic error from various sources possible. In particular, a large contributor to the overall error budget of the previous calculation is the systematic error from excited state pollution (or, equivalently, the dependence of the result on the minimum fit distance used). In the previous 

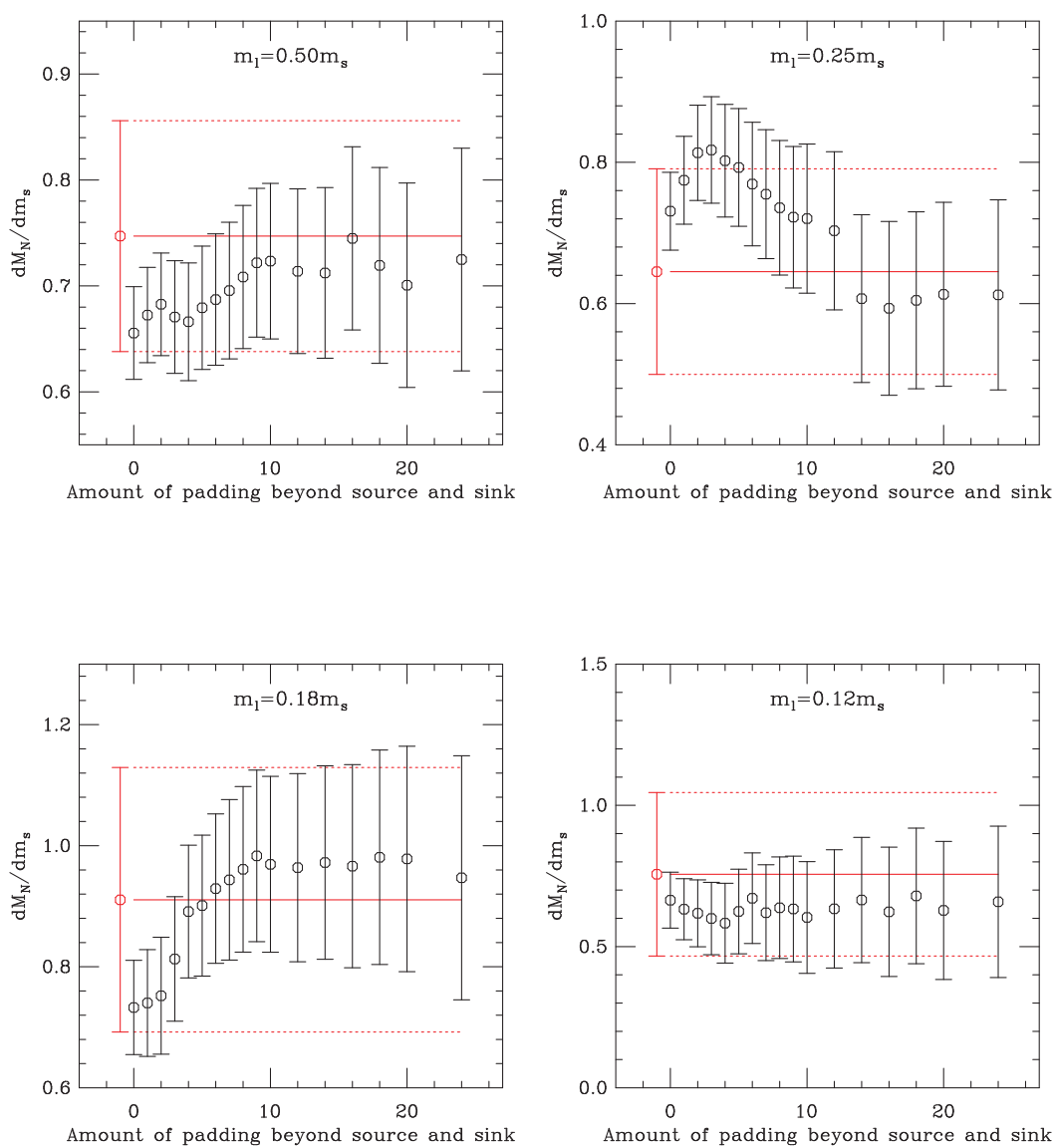

Figure 2: The effect of varying the padding size for condensate measurements on $\frac{\partial M_{N}}{\partial m_{s}}$ for each of the four $a=0.12 \mathrm{fm}$ ensembles studied. A padding size of $4 a$, for instance, means that the condensate is measured in the region between propagator source and sink, and for four time units on each end. The red value and dotted lines show the result using the old method, in which the condensate is summed over the whole lattice.

calculation the statistical errors were so large for values of $d_{\min }$ larger than the chosen one that it is difficult to judge whether the dependence of $\frac{\partial M_{N}}{\partial m_{s}}$ on $d_{\min }$ is due to systematic or statistical effects. In Ref. [3] we chose to give a conservative estimate for this systematic error; with the better statistics from this method it may be possible to reduce this estimate. Here we repeat the analysis of the dependence of $\frac{\partial M_{N}}{\partial m_{s}}$ on $d_{\text {min }}$ using the new method with a pad size of $4 a$. The results are shown in Figure 4.

Three of these four ensembles, along with preliminary data from a fifth, show no strong dependence of $\frac{\partial M_{N}}{\partial m_{s}}$ on $d_{\text {min }}$, but the $m_{l}=0.2 m_{s}$ ensemble shows a significant effect. It is likely that this effect is simply a statistical fluctuation rather than an indication that our value of $d_{\min }$ creates a large systematic error, but it prevents us from confidently giving a lower estimate of the systematic error from excited states. The fifth $a=0.12 \mathrm{fm}$ ensemble, when completed, may enable a lower estimate. 


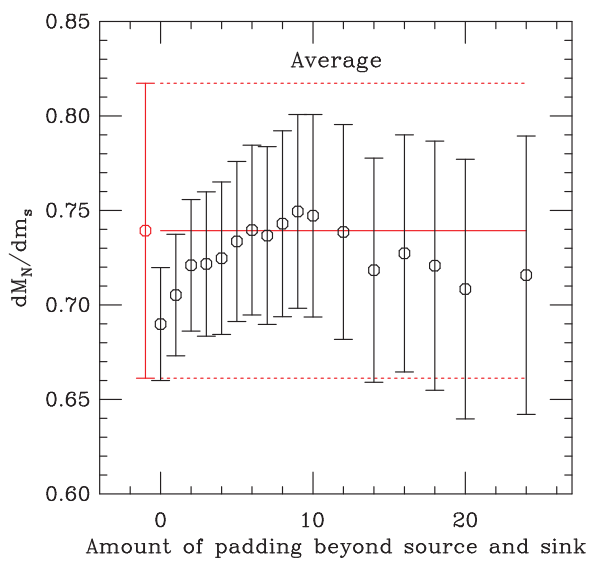

Figure 3: The effect of varying the padding size for condensate measurements on $\frac{\partial M_{N}}{\partial m_{s}}$, averaged over the four $a=0.12 \mathrm{fm}$ ensembles.
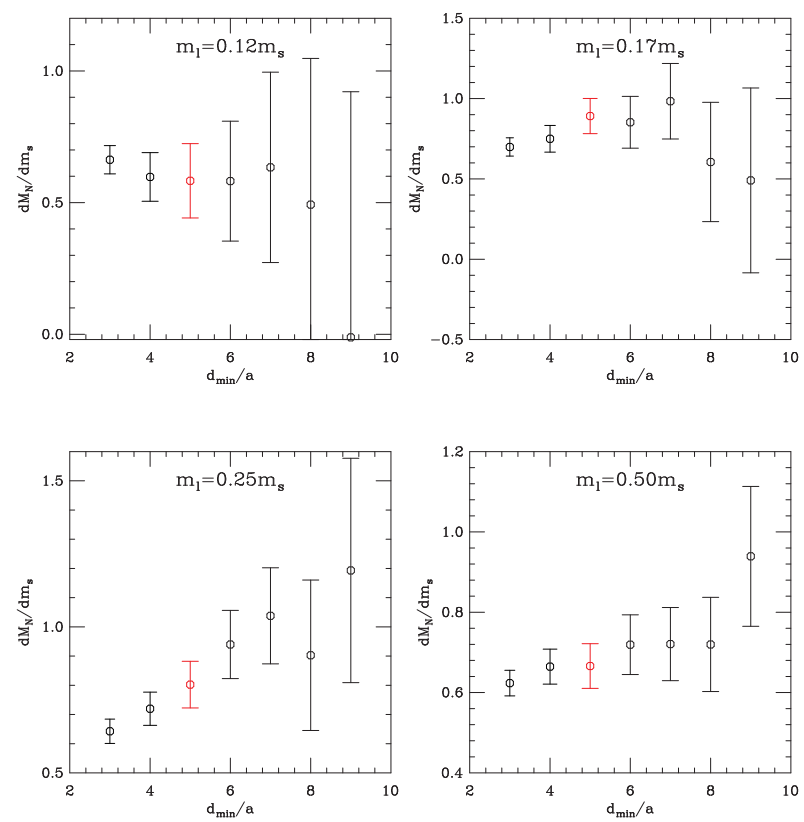

Figure 4: The dependence of $\frac{\partial M_{N}}{\partial m_{s}}$ on $d_{\min }$ for each of the four ensembles studied. The red point at $d_{\min }=5$ is the point chosen as the best balance between statistical and systematic error.

Using this procedure to improve the statistics on the finer lattice ensembles may help to improve the continuum extrapolation, which was rather poorly constrained in our previous calculation. However, the effect on the overall error budget from this will be minimal for the large amount of computer time required to redo nucleon propagators on the $a=0.09 \mathrm{fm}$ and/or $a=0.06 \mathrm{fm}$ lattices.

\section{Conclusion}

By considering only the part of the strange quark condensate that has physically relevant cor- 
relations with the nucleon propagator, the noise contributed by fluctuations in the condensate far from the propagator can be eliminated and the statistical error greatly reduced. In the four ensembles examined here, considering only the condensate within $4 a$ of the propagation region reduces the statistical error by nearly half without introducing any obvious systematic bias. The shift in the central value by $3 \%$ is explained simply by the reduction in statistical error, and these results are consistent with the previous MILC result [3].

The new data computed for this method (independent nucleon propagators for every source timeslice, and values of $\bar{s} s$ on every timeslice) can also be used for a direct evaluation of the threepoint function $\langle N|\bar{s} s| N\rangle-\langle 0|\bar{s} s| 0\rangle$. Preliminary results from this method are consistent with results obtained with the method presented here.

A new calculation of the nucleon strangeness using the improved values of $\frac{\partial M_{N}}{\partial m_{s}}$ on the $a=0.12$ ensembles presented here is in progress.

\section{References}

[1] E. Baltz, M. Battaglia, M.E. Peskin and T. Wizansky, Determination of Dark Matter Properties at High Energy Colliders, Phys. Rev. D 74 (2006) 103521 [hep-ph/ 0602187$].$

[2] J. Ellis, K. Olive and C. Savage, Hadronic Uncertainties in the Elastic Scattering of Supersymmetric Dark Matter, Phys. Rev. D 77, (2008) 065026; [arXiv: 0801.3656 ].

[3] D. Toussaint and W. Freeman, Strange Quark Condensate in the Nucleon in 2+1 Flavor QCD, Phys. Rev. Lett. 103, 2009, 122002 [hep-lat/0905.2432].

[4] G. Bali, S. Collins, and A. Schäfer, Strangeness and charm content of the nucleon, in proceedings of LATTICE 2009, POS (LATTICE 2009) 149; [arXiv:0911.2407].

[5] H. Ohki et al., Nucleon sigma term and strange quark content in 2+1-flavor QCD with dynamical overlap fermions, in proceedings of LATTICE 2009, POS (LATTICE 2009) 124; [arxiv:0910.3271v1].

[6] R.D. Young and A.W. Thomas, Octet baryon masses and sigma terms from an SU(3) chiral extrapolation, R. Young, private communication; [arXiv:0901.3310].

[7] H. Ohki et al., Calculation of the nucleon sigma term and strange quark content with two flavors of dynamical overlap fermions, Phys. Rev. D 78 (2008) 054502 [arXiv: 0806.4744 ].

[8] G. Bali, S. Collins and A. Schafer, in proceedings of LATTICE 2008, POS (LATTICE 2008) 161, [arXiv:0811.0807].

[9] M. Engelhardt, Strangeness in the nucleon from a mixed action calculation, in proceedings of LATTICE 2010, PoS (Lattice 2010) 137

[10] K. Takeda et al., Nucleon strange quark content in 2+1 flavor QCD in proceedings of LATTICE 2010, PoS (Lattice 2010)160

[11] A. Ramos, Sigma term and strange content of the nucleon in proceedings of LATTICE 2010, PoS(Lattice 2010)102

[12] C. Jung and T. Izubuchi, trange quark content of nucleon via reweighting on (2+1)-flavor domain wall fermion lattices in proceedings of LATTICE 2010, PoS (Lattice 2010) 111

[13] MILC: A. Bazavov et al., Full nonperturbative QCD simulations with $2+1$ flavors of improved staggered quarks Rev. Mod. Phys. 82, 1349 (2010); [arXiv: 0903.3598 ]. 